\title{
Huggable communication medium decreases cortisol levels
}

\section{SUBJECT AREAS: \\ QUALITY OF LIFE \\ PSYCHOLOGY}

Received

6 September 2013

Accepted

9 October 2013

Published

23 October 2013

Correspondence and requests for materials should be addressed to H.S. (sumioka@atr.jp)

\author{
Hidenobu Sumioka', Aya Nakae ${ }^{2}$, Ryota Kanai ${ }^{3,4}$ \& Hiroshi Ishiguro ${ }^{1,5}$
}

'Hiroshi Ishiguro Laboratory, Advanced Telecommunications Research Institute International, 2-2-2 Hikaridai, Keihanna Science City, Kyoto 619-0288, Japan, ${ }^{2}$ Department of Anesthesiology \& Intensive Care, Osaka University Graduate School of Medicine, 2-2 Yamadaoka, Suita, Osaka 565-0871, Japan, ${ }^{3}$ Institute of Cognitive Neuroscience, University College London, 17 Queen Square, London WCIN 3AR, United Kingdom, ${ }^{4}$ School of Psychology, Sackler Centre for Consciousness Science, University of Sussex, Pevensey 1, Falmer, BN1 9QH, United Kingdom, ${ }^{5}$ Department of Systems Innovation, Osaka University Graduate School of Engineering Science, 1-3 Machikaneyama, Toyonaka, Osaka 560-8531, Japan.

Interpersonal touch is a fundamental component of social interactions because it can mitigate physical and psychological distress. To reproduce the psychological and physiological effects associated with interpersonal touch, interest is growing in introducing tactile sensations to communication devices. However, it remains unknown whether physical contact with such devices can produce objectively measurable endocrine effects like real interpersonal touching can. We directly tested this possibility by examining changes in stress hormone cortisol before and after a conversation with a huggable communication device. Participants had 15-minute conversations with a remote partner that was carried out either with a huggable human-shaped device or with a mobile phone. Our experiment revealed significant reduction in the cortisol levels for those who had conversations with the huggable device. Our approach to evaluate communication media with biological markers suggests new design directions for interpersonal communication media to improve social support systems in modern highly networked societies.

overs cuddle and hug. A nurse strokes a patient's shoulder to reduce pain. An exhausted office worker gets a massage from a therapist for stress relief. In human interactions, interpersonal touch plays an important role in governing our emotional and physical well-being ${ }^{1,2}$. Its effects have been widely reported: it conveys emotions $^{3}$, reduces pain ${ }^{4}$, enhances a person's tipping behavior and compliance known as the "midas touch effect" ${ }^{35,6}$, and positively evaluates the person who initiated the touch ${ }^{7,8}$. Its therapeutic effects on physical and mental stress have been supported by a large body of evidence ${ }^{2,9}$.

Early studies showed the psychological effects of interpersonal touch (e.g. $\left.{ }^{7}\right)$, but recent studies have started to assess its physiological effects ${ }^{10}$. For example, such positive physical contact as hugging and massages from partners reduces cortisol, increases oxytocin, and lowers systolic blood pressure in stressful situations ${ }^{11-14}$. Such effects are also observed in physical contact from strangers or animals, as shown by studies on the psychological effects of massages ${ }^{15,16}$ and animal-assisted therapy ${ }^{17}$.

In technology-mediated remote communications, tactile stimulation is critically absent despite the recent expansion of online communication tools that are typically limited to text-based messaging such as emails and instant messengers or video-based communication like Skype and Google Hangout. In recognizing this limitation of current communication tools, researchers on communication media have attempted to introduce physical contact to communication devices to achieve the psychological effects brought by interpersonal touch ${ }^{18}$ and to facilitate social interactions with robots that assist people in everyday life $\mathrm{e}^{19-21}$.

For example, several state-of-the-art communication devices have been designed to add physical contact to the Internet and online long-distance communication between couples, friends, and family members. "Hug-Shirt," which was awarded as one of the Best invention of 2006 by Time magazine, conveys the sensation of a hug to a distant person. Another haptic stimulation system called TapTap can record and play human touches, allowing the broadcasting of interpersonal touch to distant people ${ }^{22}$. Such wearable devices offer the possibility of virtual tactile stimulation with vibration, and other studies created the sense of physical contact by developing robotic avatars that represent distant people ${ }^{23,24}$ or combining wearable interfaces as an effector and a physical object as an input device $e^{25}$.

Although these devices have been designed on the premise that the touches mediated by them would produce similar effects as those expected in real human-to-human interactions, there have been no direct empirical tests of 
their physiological impact. The critical question remains unanswered: do tactile communication devices induce physiological and behavioral responses like real interpersonal touches ${ }^{1,18}$ ?

The psychological and behavioral effects of physical contact with artificial systems have been shown for touches from interactive robots. A series of field studies with animal-like robots showed that long-term interactions with robots have positive psychological effects on elderly people $e^{26-28}$. One study reported that touches initiated by a robot motivate humans in terms of the number of working actions and the amount of working time for a monotonous $\operatorname{task}^{29}$.

Even though these studies indicate that physical touching by robots has psychological and behavioral effects, it remains unclear whether the artificial realization of interpersonal touch can produce physiological responses as observed in real human interactions. To address this issue, we investigated whether endocrine changes are observed following a brief conversation through a huggable communication device. This approach allowed us to quantitatively evaluate the physiological effects of the mediated touch without relying on subjective reports of affective states.

We hypothesized that communication with a remote person by giving a hug to a physical device would be sufficient to influence the human neuroendocrine system. To test this idea, we examined the changes in cortisol hormone, which is a reliable biomarker of psychological illnesses ${ }^{30}$, before and after participants engaged in a human-human conversation mediated by a huggable communication device. We focused on the cortisol hormone because stress relief is one of the most critical issues in providing social support to facilitate recovery from many types of mental and physical problems ${ }^{9}$. Considering the potential applications of communication media for social support, the impact of the media on stress relief is highly relevant.

In the present study, participants had a conversation with a stranger while hugging a human-shaped cushion called Hugvie ${ }^{\circledR 31}$ that functioned as a mobile phone (Hug group). In a control group, participants went through the same procedure, but used a mobile phone instead of Hugvie (Phone group) (Figure 1). To assess the neuroendocrine responses to the social interaction with the communication media, we measured cortisol levels before and after the conversation session. We collected the cortisol levels both from the blood and salivary samples since they can be dissociated due to differences in their regulatory mechanisms ${ }^{30}$. We predicted that physical contact with the huggable device would reduce the cortisol levels at a greater rate than the control group in which participants had conversations on a mobile phone without physical contact. Finally, we also evaluated the effects of physical contact on subjective psychological states with a post-session questionnaire that assessed positive affect, negative affect, and calmness.

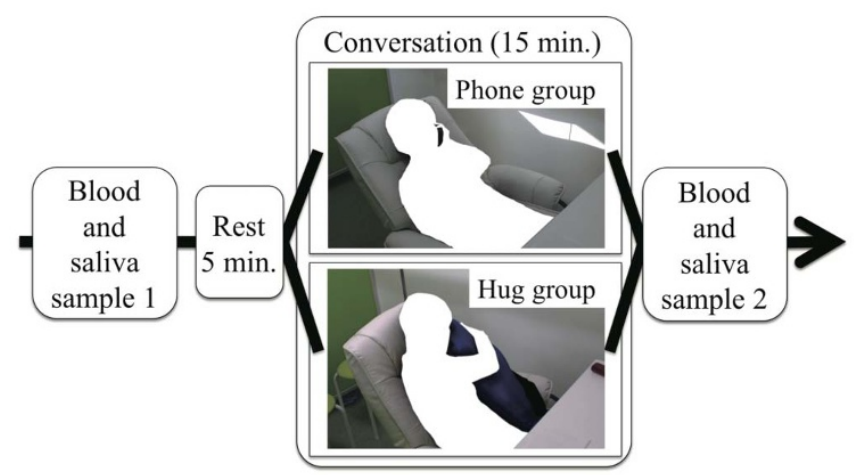

Figure 1 | Experimental paradigm.

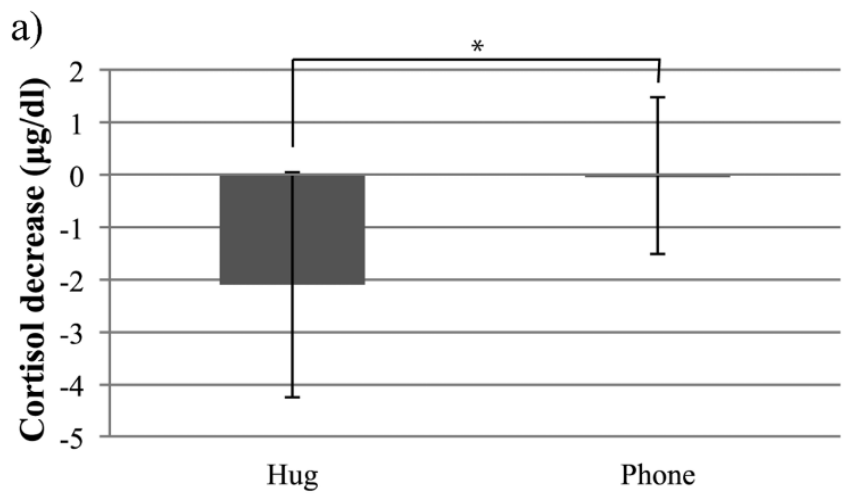

b)

\section{Condition}

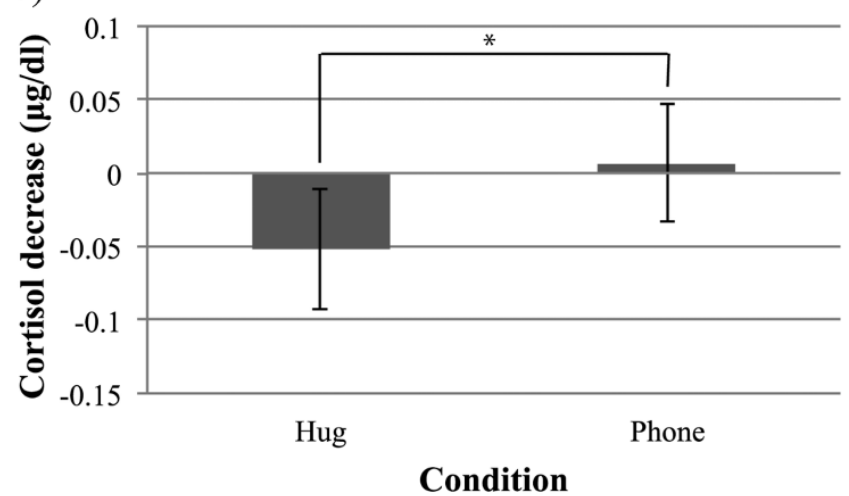

Figure 2| Mean decreases in blood (a) and saliva (b) cortisol by subtraction of cortisol levels before conversation from those after. Error bars represent standard deviation.

\section{Results}

As we predicted, the cortisol changes in the blood revealed that the participants in the Hug group showed a significantly greater decrease of cortisol after the conversations than those in the Phone group $(t=$ 2.26, $p<0.05$, ES: $d=1.13$ ) (Figure $2(\mathrm{a})$ ). We also found a significant reduction of cortisol in the saliva $(t=2.9, p<0.05$, ES: $d=1.45)$ (Figure 2 (b)). The decrease of the blood cortisol levels was correlated with that of the saliva cortisol $(r=0.54, p<0.05)$ (Figure 3$)$. We also confirmed strong correlations between the saliva and blood cortisol levels in all the samples $(r=0.82, p<0.001)$, in the samples collected before the conversations $(r=0.84, p<0.001)$, and those collected after $(r=0.79, p<0.001)$. The consistencies between the saliva and blood cortisol levels corroborate the reliability of our measures.

We found no significant differences in the participant positive affect, negative affect, and calmness between the groups (positive

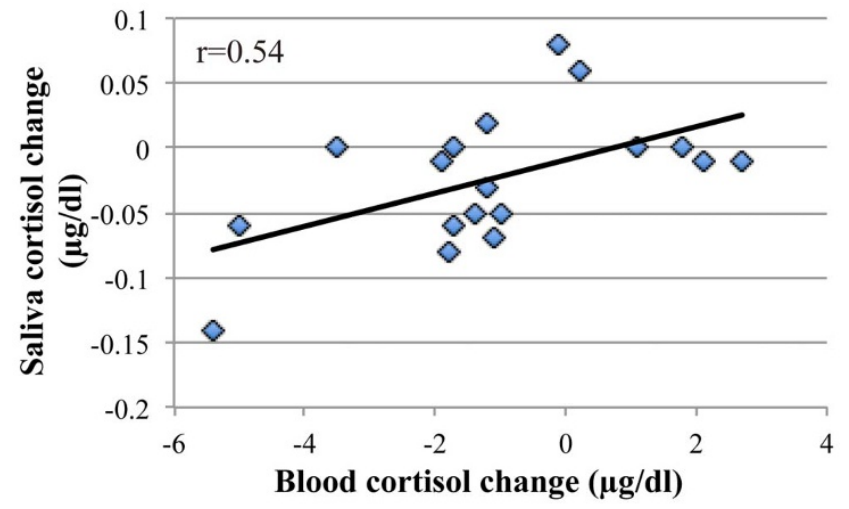

Figure 3 Correlation between cortisol changes in blood and saliva. 
affect: $t=0.38, p=0.71$, ES: $d=0.20$; negative affect: $t=1.7, p=$ 0.11 , ES: $d=0.86$; calmness: $t=2.0, p=0.07$, ES: $d=1.01$ ) (Figure 4). These subjective reports of affective states were not correlated with the cortisol levels after the conversation. Blood cortisol levels were not correlated with positive affect $(r=0.36, p=0.16)$, negative affect $(r=-0.01, p=0.96)$, or calmness $(r=0.21, p=$ $0.41)$. The saliva cortisol levels were not also correlated with negative affect $(r=-0.27, p=0.30)$ or calmness $(r=0.23, p=0.38)$, although a weak correlation was found in positive affect $(r=0.42$, $p=0.096)$.

\section{Discussion}

We found that hugging a huggable communication medium reduces the cortisol levels in both saliva and blood. These results, which support our hypothesis that physical contact with communication media can produce an effect even at the endocrine level, suggest that physical contact with such a medium might be effectively used for mental stress relief. To the best of our knowledge, this is the first study that demonstrated an endocrine effect from physical contact with a communication medium.

We also found a reduction of the cortisol levels both in the blood and saliva samples and a positive correlation between the changes in salivary and blood cortisol. This indicates that we can use salivary cortisol, which can be more easily handled than blood cortisol, to evaluate the effect of physical touch with communication media. We expect salivary cortisol to be a promising new measure to assess the effects of physical touch with communication media that have been evaluated with behavioral or psychological measures.

Our results provide two important implications. First, they suggest that communication media do not need to actively stimulate a person's skin to reduce cortisol levels. In previous research of interpersonal touching, active touching by others, such as stroking arms and massaging, was primarily used as tactile stimuli ${ }^{10,11,14}$. Other studies used a combination of several types of inactive touch, such as holding hands and hugging, and other factors (e.g., watching romantic videos) ${ }^{12,13}$. Little investigation has been conducted on the endocrine effect of single inactive touch except a study that reported the changes in the heart rate and blood pressure during gentle touches of the wrist ${ }^{32}$. Our results demonstrate that a 15-minute inactive touch with an inanimate object reduces the level of stress hormone.

Second, our results indicate that communication media can be used as research tools to investigate the positive effects of physical touch independently of the touching situation and the toucher. The effects of interpersonal touch on physiological responses are affected by how people are touched and by whom ${ }^{33}$. For example, while positive physiological changes are induced by a hug with a friend or family member, such changes do not occur for a hug with a stranger of the opposite sex because it could be taken as sexually

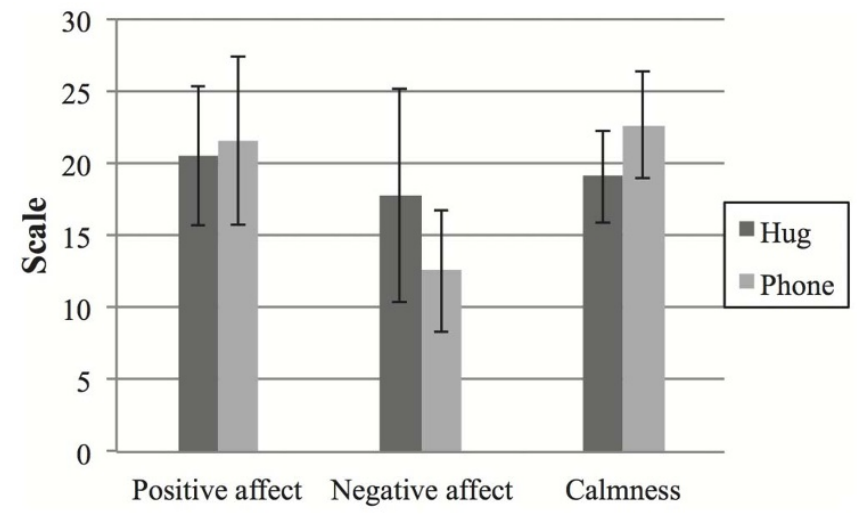

Figure $4 \mid$ Mean scores of general affect scale. Error bars represent standard deviation. offensive. By contrast, our present study shows that hugging an inanimate object reduces cortisol even during conversations with a stranger of the opposite sex. This suggests that communication media allow us to separate the actual effects of physical contact from the effect of intimate relationships in interpersonal touching, which could induce multiple effects.

In contrast to the cortisol results, the participant's subjective affect showed no significant difference between both groups. This suggests that the intervention with the huggable device did not induce subjectively perceptible psychological changes despite the objectively measurable changes in the endocrinological states. Interestingly, similar results have been reported in a previous study on real interpersonal touches. Ditzen et al. examined how physical touch by an intimate partner mitigates the stress response and found that women who received physical contact from their partners exhibited significantly lower cortisol levels and heart rates in responses to stress than those who received verbal social support or no social interaction ${ }^{11}$. However, despite the physiological differences in the cortisol levels, they found no significant differences in the self-reports of psychological states. These results suggest that subjective reports of mental states may not be as sensitive as physiological measures such as cortisol levels, because we have only limited ability to introspect our own physiological states ${ }^{34}$.

The ability to reduce cortisol levels seems suitable for improving the quality of intimate social interaction in which trust and bonding are crucial. For example, remote counseling services are widely used to improve patients' psychological states and mental health. The quality of communication with therapists may be enhanced by huggable devices, since they are typically conducted with telephones, internet, or videophones ${ }^{35}$.

Which aspects of hugging the cushion-like, huggable device are critical for cortisol reduction? We believe at least two elements warrant further consideration: the posture while using the device and the quality of the tactile sensation from it. First, the pose itself can cause psychological, physiological, and behavioral changes. For example, Carney et al. showed that a high-power pose (taking up more space and keeping limbs open) elevates the dominance hormone testosterone and reduces cortisol more than a low-power pose (taking up less space and keeping legs closed ${ }^{36}$. The hugging posture itself may have influenced the cortisol levels. Future studies should explore the influences of other postures. Second, the tactile quality of Hugvie is another element that may have caused the physiological changes. Recent neuroscience studies have shown that pleasant and light tactile stimulation is differentiated from rapid and intense tactile stimulation in neural transmissions ${ }^{37-39}$; the former is transmitted by unmyelinated tactile fibers (C-tactile fibers), and the latter by myelinated nerve fibers. Microneurography studies have suggested the possibility that C-tactile fibers in humans innervate the hairy skin but not glabrous skin ${ }^{40}$. Their innervation density is still not assessed in detail due to the limitation of current techniques. But, recent studies in mice supported this possibility, reporting that a rare subset of unmyelinated fibers, which have characteristics similar to human C-tactile fibers, exclusively innervates hairy skin $^{41}$. They also showed that massage-like stroking activates these fibers and direct activation of those neurons produces positive behavioral valence ${ }^{42}$. This suggests a potential role of human C-tactile fibers in our positive affective state. Human C-tactile fibers may mediate the link between pleasant light touching and neuroendocrinological responses. The gentle tactile stimulation from Hugvie's soft surface may effectively stimulate the C-tactile fibers in our skin of the face and arms and mimic social touching. This idea remains a speculation; however, if stimulation of the C-tactile fiber turns out to be critical, the device's effect on stress reduction could be maximized by targeting the hairy skin. We can also infer from the characteristics of the human tactile system that a vibratory tactile stimulus, which is commonly used in tactile communication devices, would not be effective because the 
vibrations in such devices do not activate C-tactile fibers ${ }^{37}$. These physiological perspectives suggest possible design directions for tactile communication devices.

Interpersonal touch affects not only cortisol but also other hormones. Recent studies found that a 15-minute massage elevates oxytocin levels and induces altruistic behavior ${ }^{15,16}$. Oxytocin is a hormone that facilitates social bonding and trusting behavior ${ }^{43}$. Studies about interpersonal touches between couples show that oxytocin increases and cortisol decreases ${ }^{12,13}$. We speculate that physical contact with the huggable device may increase the oxytocin levels during conversations. In fact, one study reported that touching a teddy bear eases feelings of social exclusion and increases prosocial behavior, suggesting a possible increase in the oxytocin level from physical contact ${ }^{44}$. Therefore, it is interesting to investigate whether physical contact with huggable devices can also increase oxytocin levels and prosocial behavior.

Our current study has several limitations. First, our experiment focused on a particular demographic of participants and a conversation partner. We recruited only female participants, and our conversation situation was between older female participants and a younger male confederate to minimize the heterogeneity in the possible social relationships. However, gender, age, and cultural background could all influence the endocrine changes, as reported in interpersonal touching ${ }^{1}$. Our results need to be tested and replicated in other social settings. Second, as mentioned above, since the huggable device's texture and shape might have contributed to the hormonal changes, any generalizations should be made with caution until our findings are replicated in different conditions.

In summary, our study demonstrated that physical contact with a simple, inanimate object decreases the cortisol levels in both saliva and blood. Our findings increase understanding of the underlying mechanism of interpersonal touch and will inspire design directions of future communication devices to improve human well-being.

\section{Methods}

Subjects. To minimize the effects of menstrual hormonal cycles and gender differences in hormonal systems, we recruited eighteen relatively old, healthy female volunteers (mean age 63.7 years, S.D. 5.0) from local cities in Osaka to evaluate our novel communication devices. Subjects on medication, or suffering from acute or chronic hormonal dysregulations, psychosomatic, or psychiatric diseases were excluded. Before the study, participants were informed of the following prohibitions: 1) no alcohol or cigarettes one day before the study; 2) no exercise, eating, or drinking one hour before the study. Participants were randomly separated into two groups: the Hug group (mean age 62.0 years, S.D. 5.2), whose nine participants talked with another person while holding a huggable communication device called Hugvie, or the Phone group (mean age 65.4 years, S.D. 4.1) that talked on a communication device resembled a mobile phone. They did not know to which group they would be assigned until the devices were given. All participants gave written informed consent prior to participation. This study was approved by the ethics committee of Osaka University (Osaka, Japan) and the Advanced Telecommunications Research Institute International (Kyoto, Japan).

Conversation partner. We selected a conversation partner from candidates who applied for an experiment assistant job. In the job interviews, they exhibited their skills in a mock 10-minute conversation that followed the same procedure as in the main experiment. After a careful selection process, we chose a 22 -year-old male university student as a competent conversation partner. He was not aware of the purpose of the experiment throughout the study. He also gave informed consent and was covered under the ethical approval described above.

Communication devices. We used a human-shaped cushion phone called Hugvie (75 $\mathrm{cm}$ high and $600 \mathrm{~g}$ ) to provide tactile stimulation for the participants. Its design was inspired by observation of field studies with Telenoid, a human-like robotic medium with minimal human appearance characteristics ${ }^{24}$. Studies reported that physical contact with a Telenoid, especially hugging it, is a primary form of interaction and has strong psychological impact on a wide age range of users. Based on this finding, Hugvie was designed as a communication device that focuses on the hugging experience. It is a soft cushion filled with polystyrene microbeads and covered with spandex fiber, which is often used for microbead pillows. It resembles person opening its arms for a hug and enables us to bring the hug experience into telecommunication by putting a hands-free mobile phone inside a pocket of its "head". Since the phone is in the pocket, people can call and talk while hugging the Hugvie, increasing the feeling they are actually hugging a distant conversation partner. A
Hugvie does not have any actuators inside it. Therefore we can investigate the effect of inactive touches from it.

To maintain stable audio communication, we used a portable Bluetooth speaker (MOT-EQ5, MOTOROLA) to transmit audio information from the conversation partner instead of a standard mobile phone. This speaker was used in the Phone group as the communication device.

Experimental environment. The experimental room included two adjacent subrooms that were separated by 1.62-meter-high walls; a waiting room was used for the blood and saliva sampling and the informed consent, and another room was used for the conversation task and answering questionnaires. During the conversations and the questionnaires, participants were left alone in the conversation room and an experimenter waited in the waiting room. The conversation partner waited in an operation room located on another floor. The operation and conversation rooms were wired with RCA cables to maintain stable audio communication. The audio information in the conversation room was carried to the operation room by a hidden microphone. Conversations were video-recorded with the agreement of the participants.

Experimental procedure. The experiments were conducted in the morning and the afternoon (10:00-12:00 and 13:00-15:00). To avoid the influence of diurnal rhythms on hormones, the assignments into two groups were counterbalanced for morning and afternoon sessions.

The experiment consisted of four phases (Figure 1). Participants were first led to a waiting room. After filling out pre-conversation questionnaires about their frequency of internet surfing, knowledge of and attitudes to interactive robots, and about themselves, saliva and blood samples were taken from all participants (Sample 1). Each participant gave a saliva sample by a cylindrical plain cotton roll, and a licensed technician drew $8 \mathrm{ml}$ of blood from an antecubital or dorsal hand vein.

After the first sample, the participants were led to the conversation room next to the waiting room where they relaxed on a reclining easy chair for 5 minutes. Then the experimenter gave Hugvie to them in the Hug group and explained how to hold it (see Supplementary Fig. S1 online); participants in the Phone group were given Bluetooth speaker. All participants were asked to have a 15-minute conversation with another male participant on the given communication device. In reality, the experiment assistant conversed with them. The participants and the assistant neither know nor see each other throughout the experiment.

At the beginning of the conversation, the assistant first introduced himself to a participant after greeting and giving his name, and asked the participant's name if she did not inform him of it. Then, he asked her best memories in the last year. $62.5 \%$ of all participants talked about their travel and the rest talked about their hobby, family, pet, and success of surgery. During the conversation, the participant and the assistant were able to talk about themselves or ask questions to the opponent freely except that the assistant was forbidden to ask participants about their education background, parental job, and political affiliation. The conversation went smoothly without any period of silence since the assistant encouraged the conversation by changing a topic, talking about himself, and asking new questions.

When the 15-minute conversations ended, the experimenter led the participants to the waiting room for the second saliva and blood samples (Sample 2) that were promptly taken in the same manner as the first one. Next the participants returned to the conversation room and joined another experiment where they played a computer game for 10 minutes. Finally, they answered a post-conversation questionnaire about their affective states during the conversation and then they were debriefed before being dismissed.

Cortisol collection and analysis. The saliva and blood samples were assayed for cortisol determination by a radio immunoassay (RIA; Immunotech Inc., Quebec, Canada) using a standard curve method with reported detection limits of $0.17 \mu \mathrm{g} / \mathrm{dl}$. The cross-reactivity of the assay was $8.4 \%$ with corticosterone, $1.5 \%$ with cortisone $<0.1 \%$ with predonisone, and $<0.1 \%$ with other naturally occurring steroids. For the saliva assay, thawed samples were centrifuged at 3,000 rpm for ten minutes and stored until analysis at $-80^{\circ} \mathrm{C}$. Plasma samples were obtained within 30 minutes after collection in plastic tubes containing EDTA as an anticoagulant, centrifuged at $3,000 \mathrm{rpm}$ for ten minutes, and stored until analysis at $-80^{\circ} \mathrm{C}$. All the samples from the participants were included in the same assay batch to eliminate within subject inter-assay variance. All the samples were assayed in duplicate and averaged. The inter-assay coefficients of variance were $3.5 \%$

The cortisol levels in several saliva samples were below the lower limit of measurement $(0.06 \mu \mathrm{g} / \mathrm{dl})$. Therefore, we set them to zero and subtracted the limit from the cortisol value of other saliva samples. This procedure was not applied for the blood samples because cortisol hormones were obtained above the lower limit in all the samples. The effect of physical touch was measured as the decrease of cortisol that was calculated by subtracting the cortisols before the conversation from those after the conversation (Sample 2 - Sample 1). We used a t-test to evaluate the difference of the cortisol decrease in the saliva and the blood between the two groups because the normality and equal variance of the saliva and blood data between the two groups were confirmed with Shapiro-Wilk normality test and F test, respectively (see Supplemental Information). Pearson's product-moment correlation was used to calculate the correlation between the saliva and blood samples.

Affective states. We asked the participants their affect in the post-conversation questionnaire. Positive and Negative Affect Schedule (PANAS) is a well-known scale 
to capture temporary positive and negative affect ${ }^{45}$. However, it does not include such deactivation pleasant feelings such as calmness and relaxation that are also related to mental stress ${ }^{46}$. Therefore, we used a Japanese scale called the General Affect Scale $(\mathrm{GAS})^{47}$, which is based on PANAS and other Japanese affect scales, to evaluate positive affect, negative affect, and calmness. This 24 -adjective questionnaire, which was answered on a scale from 1 (not at all) to 4 (very), measures each affective factor by adding the ratings of eight corresponding adjectives (see Supplementary Table S1 online). We applied t-tests and Pearson's product-moment correlation for analysis because of the normality and the equal variance of all the data (see Supplemental Information). Since we could not collect GAS responses from one participant in the Hug group due to system failure during the questionnaire, we used responses from the other eight participants in our analysis related to GAS.

1. Gallace, A. \& Spence, C. The science of interpersonal touch: An overview. Neurosci. Biobehav. Rev. 34, 246-259 (2010).

2. Field, T. Touch for socioemotional and physical well-being: a review. Dev. Rev. 30, 367-383 (2010)

3. Hertenstein, M. J., Holmes, R., McCullough, M. \& Keltner, D. The communication of emotion via touch. Emotion 9, 566-573 (2009).

4. Kutner, J. S. et al. Massage therapy versus simple touch to improve pain and mood in patients with advanced cancer: a randomized trial. Ann. Intern. Med. 149, 369-379 (2008)

5. Crusco, A. H. \& Wetzel, C. G. The midas touch: the effects of interpersonal touch on restaurant tipping. Pers. Soc. Psychol. B. 10, 512-517 (1984).

6. Guéguen, N. \& Fischer-Lokou, J. Another evaluation of touch and helping behavior. Psychol. Rep. 92, 62-64 (2003).

7. Fischer, J. D., Rytting, M. \& Heslin, R. Hands touching hands: affective and evaluative effects of interpersonal touch. Sociometry 39, 416-421 (1976).

8. Erceau, D. \& Guéguen, N. Tactile contact and evaluation of the toucher. J. Soc. Psychol. 147, 441-444 (2007)

9. Cohen, S. Social relationships and health. Am. Psychol. 59, 676-684 (2004).

10. Field, T., Hernandez-Reif, M., Diego, M., Schanberg, S. \& Kuhn, C. Cortisol decreases and serotonin and dopamine increase following massage therapy. Int. J. Neurosci. 115, 1397-1413 (2005).

11. Ditzen, B. et al. Effects of different kinds of couple interaction on cortisol and heart rate responses to stress in women. Psychoneuroendocrinology 32, 565-574 (2007).

12. Light, K. C., Grewen, K. M. \& Amico, J. A. More frequent partner hugs and higher oxytocin levels are linked to lower blood pressure and heart rate in premenopausal women. Biol. Psychol. 69, 5-21 (2005).

13. Grewen, K. M., Girdler, S. S., Amico, J. \& Light, K. C. Effects of partner support on resting oxytocin, cortisol, norepinephrine, and blood pressure before and after warm partner contact. Psychosom. Med. 67, 531-538 (2005).

14. Holt-Lunstad, J., Birmingham, W. A. \& Light, K. C. Influence of a "warm touch" support enhancement intervention among married couples on ambulatory blood pressure, oxytocin, alpha amylase, and cortisol. Psychosom. Med. 70, 976-985 (2008)

15. Morhenn, V. B., Park, J. W., Piper, E. \& Zak, P. J. Monetary sacrifice among strangers is mediated by endogenous oxytocin release after physical contact. Evol. Hum. Behav. 29, 375-383 (2008)

16. Morhenn, V. B., Beavin, L. E. \& Zak, P. J. Massage increases oxytocin and reduces adrenocorticotropin hormone in humans. Alternat. Ther. Health Med. 18, 11-18 (2012)

17. Beetz, A., Uvnäs-Moberg, K., Julius, H. \& Kotrschal, K. Psychosocial and psychophysiological effects of human-animal interactions: the possible role of oxytocin. Frontiers in Psychology 3, 234 doi:10.3389/fpsyg.2012.00234 (2012).

18. Haans, A. \& IJsselsteijn, W. Mediated social touch: a review of current research and future directions. Virtual Reality 9, 149-159 (2006)

19. Stiehl, W. \& Breazeal, C. Design of a therapeutic robotic companion for relational, affective touch. Proc. of the 14th IEEE Int. Workshop on Robot and Human Interactive Communication 408-415 (2005).

20. Shibata, T. An overview of human interactive robots for psychological enrichment. Proc. of the IEEE 92, 1749-1758 (2004).

21. Kanda, T., Ishiguro, H., Ono, T., Imai, M. \& Nakatsu, R. Development and evaluation of an interactive humanoid robot. Proc. of the IEEE Int. Conf. on Robotics and Automation 1848-1855 (2002).

22. Bonanni, L., Vaumobilee, C., Lieberman, J. \& Zuckerman, O. TapTap: a haptic wearable for asynchronous distributed touch therapy. Ext. Abst. on Human Factors in Comp. Sys. 580-585 (2006).

23. DiSalvo, C., Gemperle, F., Forlizzi, J. \& Montgomery, E. The Hug: an exploration of robotic form for intimate communication. Proc. of the 12th IEEE Int. Workshop on Robot and Human Interactive Communication 403-408 (2003).

24. Ogawa, K. et al. Exploring the natural reaction of young and aged person with Telenoid in a real world. J. Adv. Comp. Intelligence and Intelligent Informatics 15, 592-597 (2011).

25. Mueller, F. et al. Hug over a distance. Ext. Abst. on Human Factors in Comp. Sys. 1673-1676 (2005).

26. Wada, K., Shibata, T., Saito, T., Sakamoto, K. \& Tanie, K. Psychological and social effects of one year robot assisted activity on elderly people at a health service facility for the aged. Proc. of the IEEE Int. Conf. on Robotics and Automation $2785-2790$ (2005).
27. Wada, K., Shibata, T., Saito, T. \& Tanie, K. Effects of robot-assisted activity for elderly people and nurses at a day service center. Proc. of the IEEE 92, 1780-1788 (2004).

28. Wada, K. \& Shibata, T. Robot therapy in a care house - Its sociopsychological and physiological effects on the residents. Proc. of the IEEE Int. Conf. on Robotics and Automation 3966-3971 (2006).

29. Nakagawa, K. et al. Effect of robot's active touch on people's motivation. Proc. of the 6th ACM/IEEE Int. Conf. on Human-robot Interaction 465-472 (2011).

30. Hellhammer, D. H., Wüst, S. \& Kudielka, B. M. Salivary cortisol as a biomarker in stress research. Psychoneuroendocrinology 34, 163-171 (2009).

31. Minato, T., Nishio, S. \& Ishiguro, H. Evoking an affection for communication partner by a robotic communication medium. Demo. Session Proc. of the 8th ACM/IEEE Int. Conf. on Human-Robot Interaction D07 (2013).

32. Edens, J. L., Larkin, K. T. \& Abel, J. L. The effect of social support and physical touch on cardiovascular reactions to mental stress. J. Psychosom. Res. 36, 371-381 (1992).

33. Nilsen, W. J. \& Vrana, S. R. Some touching situations: The relationship between gender and contextual variables in cardiovascular responses to human touch. Ann. Behav. Med. 20, 270-276 (1998).

34. Critchley, H. D., Wiens, S. S., Rotshtein, P., Öhman, A. \& Dolan, R. Neural systems supporting interoceptive awareness. Nat. Neurosci. 7, 189-195 (2004)

35. Bee, P. et al. Psychotherapy mediated by remote communication technologies: a meta-analytic review. BMC Psychiatry 8, 60 doi:10.1186/1471-244X-8-60 (2008).

36. Carney, D. R., Cuddy, A. J. C. \& Yap, A. J. Power posing brief nonverbal displays affect neuroendocrine levels and risk tolerance. Psych. Sci. 21, 1363-1368 (2010).

37. Olausson, H. et al. Unmyelinated tactile afferents signal touch and project to insular cortex. Nat. Neurosci. 5, 900-904 (2002).

38. Löken, L. S., Wessberg, J., McGlone, F. \& Olausson, H. Coding of pleasant touch by unmyelinated afferents in humans. Nat. Neurosci. 12, 547-548 (2009).

39. McGlone, F. \& Reilly, D. The cutaneous sensory system. Neurosci. Biobehav. Rev. 34, 148-159 (2010).

40. Olausson, H., Wessberg, J., Morrison, I., McGlone, F. \& Vallbo, Å. B. The neurophysiology of unmyelinated tactile afferents. Neurosci. Biobehav. Rev. 34, 185-191 (2010)

41. Liu, Q. et al. Molecular genetic visualization of a rare subset of unmyelinated sensory neurons that may detect gentle touch. Nat. Neurosci. 10, 946-948 (2007).

42. Vrontou, S., Wong, A. M., Rau, K. K., Koerber, R. H. \& Anderson, D. J. Genetic identification of $\mathrm{C}$ fibres that detect massage-like stroking of hairy skin in vivo. Nature 493, 669-673 (2013).

43. Bartz, J. A., Zaki, J., Bolger, N. \& Ochsner, K. N. Social effects of oxytocin in humans: context and person matter. Trends Cogn. Sci. 15, 301-309 (2011).

44. Tai, K., Zheng, X. \& Narayanan, J. Touching a teddy bear mitigates negative effects of social exclusion to increase prosocial behavior. Soc. Psychol. Person. Sci. 2, 618-626 (2011).

45. Watson, D., Clark, L. A. \& Tellegen, A. Development and validation of brief measures of positive and negative affect: the PANAS scales. J. Pers. Soc. Psychol. 54, 1063-1070 (1988)

46. Russell, J. A. \& Barrett, L. F. Core affect, prototypical emotional episodes, and other things called emotion: dissecting the elephant. J. Pers. Soc. Psychol. 76, 805-819 (1999).

47. Ogawa, T., Monchi, R., Kikuya, M. \& Suzuki, N. Development of the general affect scales. JPN J. Psychol. 71, 241-246 (In Japanese) (2000).

\section{Acknowledgments}

We thanks Y. Ishida and A. Muto for their assistance in saliva and blood collection and analysis and A. Kimura for her help with subject recruitment. HS thanks T. Minato, K. Koda, K. Kuwamura, and K. Sakai for their helping with the experimental setup and S. Nishio for financial support and useful advice to ethical aspect of this work. This work has been supported by JST CREST (Core Research of Evolutional Science and Technology) research promotion program "Creation of Human-Harmonized Information Technology for Convivial Society" Research Area and the Grant-in-Aid for the Global COE Program "Center of Human-friendly Robotics Based on Cognitive Neuroscience".

\section{Author contributions}

H.S., A.N. and R.K. wrote the main manuscript text. H.I. reviewed the manuscript. All authors designed research.

\section{Additional information}

Supplementary information accompanies this paper at http://www.nature.com/ scientificreports

Competing financial interests: Dr. Sumioka and Dr. Ishiguro are employed by Advanced Telecommunications Research Institute International, which has patents on Hugvies. Dr. Ishiguro has consulted for Vstone Co., Ltd., which sells Hugvies, and received compensation. He also owns stock in the company. Dr. Nakae and Dr. Kanai declare no potential conflict of interest. 
How to cite this article: Sumioka, H., Nakae, A., Kanai, R. \& Ishiguro, H. Huggable communication medium decreases cortisol levels. Sci. Rep. 3, 3034; DOI:10.1038/srep03034 (2013) cc) (i) $\odot$ This work is licensed under a Creative Commons AttributionBy No NonCommercial-NoDerivs 3.0 Unported license. To view a copy of this license, visit http://creativecommons.org/licenses/by-nc-nd/3.0 